\title{
La persona adolescente y su experiencia durante la etapa intermedia ${ }^{1}$
}

Sophía González Zúñiga Ana María Quezada Ugalde ${ }^{3}$

\author{
Institución: Colegio Ing. Samuel Sáenz Flores
}

\section{CÓMO CITAR}

González, S. y Quezada, A.. (2015). La persona adolescente y su experiencia durante la etapa intermedia. Rev. Enfermería Actual en Costa Rica, 29, 1-14. DOI: http://dx.doi.org/10.15517/revenf.v0i29.19572

\section{RESUMEN}

Introducción: La adolescencia es considerada una etapa de vulnerabilidad en la vida de todas las personas, cuya cobertura de atención dentro del sistema de salud de esta población es escasa, con un abordaje dirigido exclusivamente a las problemáticas, motivo por el que requiere mayor especialización. El presente artículo tiene como objetivo presentar el diagnóstico situacional de un grupo de adolescentes en etapa intermedia con el fin de posteriormente elaborar un programa de Enfermería Pediátrica para la atención de la persona adolescente en etapa intermedia, en una institución de enseñanza media durante los años 2013 - 2014.

Método: La metodología implementada es de tipo cualitativo con un enfoque epistemológico constructivista; el análisis de los datos se efectuó mediante la triangulación de la información recopilada.

Resultados: Entre los principales hallazgos de la fase diagnóstica se obtuvo que los adolescentes priorizaron problemáticas como el fenómeno de las drogas y situaciones relacionadas con la sexualidad; además describen su experiencia a lo largo de esta etapa de la vida, los cambios acontecidos, y la interacción con pares y familia, información necesaria para que un profesional de enfermería pediátrica intervenga de manera adecuada.

Conclusión: Se concluye que es fundamental establecer un primer contacto con la persona adolescente en función de identificar de forma conjunta sus necesidades y brindarles la oportunidad de proponer posibles respuestas. El continuo acercamiento favorece el desarrollo de la empatía con el fin de obtener mayor apertura del individuo frente a los profesionales de enfermería como una figura de acompañamiento a lo largo de su adolescencia.

Palabras claves: adolescencia, diagnóstico-necesidades, enfermería, experiencia-en-colegio.

1 Fecha de recepción: 24 de febrero del 2015

Fecha de aceptación: 26 de mayo del 2015

2 Enfermera pediatra, Coordinadora Curso de Formación ATAPS, Escuela de Enfermería. Universidad de Costa Rica. Correo electrónico: sophiawwjd@gmail.com

3 Enfermera pediatra, docente, Escuela de Enfermería. Universidad de Costa Rica. Correo electrónico: ana.QUEZADAUGALDE@ucr.ac.cr 


\title{
The adolescent and his experience during the intermediate stage ${ }^{1}$
}

Sophía González Zúñiga² Ana María Quezada Ugalde ${ }^{3}$

\author{
Institution: Colegio Ing. Samuel Sáenz Flores, Universidad de Costa Rica
}

\section{CITED}

González, S. \& Quezada, A.. (2015). The adolescent and his experience during the intermediate stage. Rev. Enfermería Actual en Costa Rica, 29, 1-14. DOI: http://dx.doi.org/10.15517/revenf.v0i29.19572

\begin{abstract}
Introduction. Adolescence is considered a vulnerable stage on all people's life. Within the health system, this population is characterized by a low coverage exclusively aimed at finding the problems, which requires more specialization. This article aims to present the situational diagnosis of a group of teenagers in the intermediate stage in order to further develop a program of Pediatric Nursing care for the adolescent in the intermediate stage, in an institution of secondary education for the years 2013-2014.
\end{abstract}

Method. The implemented methodology is qualitative with a constructivist epistemological approach; triangulating the information gathered performed data analysis.

Results. Among the main findings of the diagnostic phase was obtained teenagers prioritized issues such as the phenomenon of drugs and situations related to sexuality; also describe their experience along this stage of life, the changes that occurred, and interaction with peers and family, information necessary for a pediatric nurse practitioner intervene appropriately. The main findings on the diagnose phase show that adolescents prioritized issues like drugs and situations related to sexuality. They also describe their experience through this life stage, all the changes occurred and the interaction with partners and family.

Conclusion. The study reveals that it is essential to establish a first contact with the adolescent to identify all their needs in an integral form, and to give them the opportunity to propose possible solutions. Being continuously in contact stimulates the development of empathy in order to obtain a greater openness from the individual to the

1 Date of receipt: February 24, 2015

Date of acceptance: May 26, 2015

2 Nurse, Training Course for ATAPS Coordinator, School of Nursing. University of Costa Rica. Costa Rica.E-mail: sophiawwjd@gmail.com

3 Nurse. Professor of the School of Nursing. University of Costa Rica. Costa Rica. E-mail: ana.quezadaugalde@ucr.ac.cr 


\section{Revista Electrónica Enfermeria Actual en costa Rica}

nurse-practitioner as an accompaniment figure throughout their adolescence.

Key words: adolescence, nursery, needs-assessment, experience, high-school.

\section{INTRODUCCIÓN}

La protección y cuidado para la población adolescente se inició en 1924 con la Declaración de Ginebra sobre los Derechos del Niño, la cual corresponde a un primer intento de proteger de forma especial a la persona menor de 18 años; posteriormente, la Organización de las Naciones Unidas llevó a cabo la Convención sobre los Derechos del Niño en 1989.

En el caso específico de Costa Rica, no fue hasta 1998 cuando la Asamblea Legislativa emitió el Código de la Niñez y la Adolescencia como marco jurídico mínimo para la protección integral de los derechos de las personas menores de edad, en el cual se establece los principios básicos de la participación social y de procesos administrativos y judiciales que involucren el cumplimiento de los derechos y los deberes de esta población (Asamblea Legislativa de la República de Costa Rica, 2001). Además, el país cuenta con la Política Nacional para la Niñez y Adolescencia 2009-2021 creada por la UNICEF, el Patronato Nacional de la Infancia y el Consejo Nacional de la Niñez y la Adolescencia.

Las políticas mencionadas tienen como fin defender los derechos de todos los adolescentes en un país, población que -según el Fondo de las Naciones Unidas para la Infancia (2011)- ha aumentado desde la década de los 50. En relación con el dato anterior, a nivel mundial se calcula que hay un aproximado de 1200 millones de jóvenes con edades entre los 10 y 19 años, lo cual corresponde a un 19\% de la población mundial; de igual forma, en Costa Rica, del total de la población costarricense, 4301712 de personas, el 18.4\% corresponde a adolescentes entre los diez y los diecinueve años de edad (Instituto Nacional de Estadísticas y Censos, 2011).

Respecto de la atención en salud de dicho sector, la Caja Costarricense de Seguro Social (CCSS) plantea que para el año 2012 se alcanzó únicamente un 30\% de cobertura de personas mayores de diez años, lo cual podría relacionarse con la particularidad de que -desde el punto de vista biológico- los adolescentes presentan un bajo riesgo de enfermarse, razón por el que no asisten demasiado a los centros de salud. Con base en lo anterior, se destaca la urgente necesidad del país en cuanto a mejorar los niveles de cobertura para este grupo etario, a través de las estrategias de promoción de la salud y prevención de la enfermedad, en el primer nivel de atención.

En relación con dicho requerimiento, se seleccionó el escenario educativo para obtener un mayor impacto, accesibilidad, acompañamiento y seguimiento de la persona adolescente, además para enfatizar el proceso de crecimiento y desarrollo en condiciones de salud, entendida esta como "un fenómeno con implicaciones sociales, económicas y políticas y, sobre todo, un derecho fundamental" (Organización Panamericana de la Salud, 2007, p.3). De esta manera se responde a las disposiciones propuestas por el marco jurídico internacional y el costarricense en pro del resguardo de la población adolescente, además de que se reconoce las necesidades en lo que respecta a salud -en función de considerar la adolescencia como etapa de la vida única y diferente a las demás- $\mathrm{y}$, por último, se destaca que tales acciones son parte de un esfuerzo realizado por la enfermería para lograr un acercamiento entre el sistema de salud y la población adolescente en su entorno. 


\section{Revista Electrónica Enfermeria Actual en costa Rica}

En este contexto, fue gracias a la teoría de la consecución de objetivos de Imogene King, que se alcanzó el análisis e interpretación de las acciones desde la disciplina y se clarificó las interacciones entre la persona adolescente y el profesional en función del alcance de objetivos (King, 1984), máxime si se trata del área de enfermería pediátrica, dado que posee conocimientos en torno al proceso de vida de la población adolescente y la influencia de los factores físicos, psicoemocionales, sociales y epidemiológicos, domina los fundamentos pedagógicos de la educación para la salud, además, analiza su intervención para promover modelos de atención innovadores mediante la investigación y elaboración de proyectos, y enfatiza la promoción de la salud y la prevención de los procesos mórbidos (Programa de Posgrado en Ciencias de la Enfermería, s.f.) .

En relación con los antecedentes de este escrito, en el ámbito internacional se menciona a $\underline{\text { Jolly, Weiss y }}$ Liehr (2007), quienes en su investigación Understanding adolescent voice as a guide for nursing practice and research, afirman la relevancia de escuchar la voz de la población adolescente, así como saber qué es importante para ellos, con el propósito de ayudarlos a tomar decisiones positivas de salud, en medio de los desafíos que enfrentan en sus complejas vidas.

De igual forma, Campbell, Tuttle, y Knapp (2009) en The Effect of Positive Adolescent Life Skills Training on Long Term Outcomes for High-Risk Teens, demostraron que los jóvenes necesitan modelos adultos positivos para aprender a tomar decisiones saludables, rechazar los riesgos y comunicarse de manera efectiva.

También en el año 2009, Parvizy y Ahmadi, en su investigación A qualitative study on adolescence, health and family, concluyeron que se debe desarrollar una comunicación sana dentro del núcleo familiar para evitar que las situaciones familiares negativas afecten a los adolescentes, momento en el que es relevante el acompañamiento del personal de enfermería respecto de las habilidades de comunicación entre los diferentes actores, los cambios adolescentes y las tendencias sociales emergentes.

Como último antecedente internacional, está la investigación Health-risk behaviors in early adolescence, de Rew, Horner y Brown (2011), quienes afirman que la promoción de conductas protectoras en los grados escolares puede convertirse en hábitos saludables en la adolescencia, etapa durante la que el papel de enfermería es fundamental en cuanto a animar a los infantes y a sus familiares para que sigan acciones que contribuyan a la salud y bienestar general.

En el ámbito nacional, se presentan dos publicaciones de grado: en la primera,Programa de enfermería sobre educación en salud dirigido a los y las adolescentes de educación diversificada especial del Colegio Técnico Industrial de Calle Blancos Muñoz, Argüello, Arias, Gómez y Siles (2009) concluyeron que es fundamental utilizar una metodología inclusiva que considere las necesidades especiales de la persona adolescente, la influencia de la sociedad y los grupos a los cuales pertenece. El segundo trabajo, Intervención de enfermería en la adolescencia: experiencia en una institución de estudios secundarios pública, (Martínez et al, 2013) se evidencia que la educación en salud del grupo adolescente es un problema de salud pública al que los profesionales de enfermería pueden responder.

Considerando la evidencia científica descrita, este estudio correspondió a un esfuerzo investigativo desde enfermería pediátrica para acercarse a la población adolescente con una perspectiva centrada en sus necesidades y experiencias durante esta etapa con el objetivo general de desarrollar un programa de enfermería pediátrica para 


\section{Revista Electrónica Enfermeria Actual en costa Rica}

la atención de la persona adolescente en etapa intermedia en el Colegio Samuel Sáenz Flores, durante el primer semestre del año 2014.

\section{MATERIALES Y MÉTODOS}

La población estuvo integrada por las 684 personas adolescentes inscritas como estudiantes de séptimo y octavo año de secundaria en el Colegio Ing. Samuel Sáenz Flores durante el año 2013, quienes se encuentran en la etapa de adolescencia intermedia entre los 14 y 16 años de edad (González, 2010, y Retana, 2007).

De acuerdo con la capacidad operativa de recolección y análisis con la que contaron las investigadoras, y en relación con los recursos de tiempo y espacio disponibles, se determinó una muestra de tipo no probabilística, con un total de 32 personas adolescentes estudiantes de séptimo y octavo año del colegio durante el año 2013.

En cuanto a los criterios para escoger a los adolescentes se consideró que fueran estudiantes regulares del colegio en los niveles de sétimo y octavo año, que mostraran anuencia a participar en el estudio y se ubicaran en la etapa intermedia de la adolescencia.

La investigación se desarrolló desde el paradigma fenomenológico, el cual se basa en describir y entender los fenómenos (Hernández, Fernández y Baptista, 2010). De acuerdo con la función ontológica, la realidad se construyó a través del lenguaje propio de la población meta, basado en una posición epistemológica denominada constructivista ( Krause, 1995).

En cuanto al método, corresponde a investigación-acción en el que investigar e intervenir son procesos simultáneos que, en este caso, parte de las personas adolescentes, los actores principales, quienes mejor que nadie conocen sus necesidades, la estructura por modificar, el proceso por mejorar y las prácticas que requieren transformación (Hernández et al, , 2010).

Se realizó una inmersión en el contexto de la población adolescente a través de la técnica de grupos focales en la que se planteó preguntas generadoras mediante las que se recabaría las experiencias, interacciones y problemáticas existentes. De igual forma, se hizo una observación durante las actividades realizadas en la semana cívica, con el fin de identificar las relaciones adolescente-profesor y adolescente-adolescente, así como la dinámica presente en el centro educativo. Con base en lo obtenido, se construyó una categorización de las siguientes unidades de análisis: datos sociodemográficos, experiencias, interacciones y percepción.

Para el análisis de los datos, cada uno de los grupos focales fue "vaciado" en relatos codificados por colores según las unidades y subunidades de análisis previamente identificadas. Se parte de la noción de que el ser humano se encuentra en una continua interacción con el entorno que le permite generar percepciones, las cuales llegan a influir en sus interacciones y, por ende, en su salud (King, 1984). De manera que, a pesar de que el análisis es por categorías, se reconoce la estrecha relación existente entre estas, dentro de los sistemas propuestos por King, caracterizados por ser abiertos, dinámicos y estar en constante correlación.

Para lograr un primer acercamiento con la población seleccionada se realizó cuatro grupos focales con 
integrantes del mismo sexo: dos grupos con mujeres y dos con hombres. También, se llevó a cabo una observación con el fin de determinar las interacciones y el contexto en el que se desenvuelven. La población adolescente fue integrada por dos secciones de sétimo año y dos de octavo, con edades entre los 13 y 14 años.

Una vez recolectados los datos a través de las técnicas descritas, las investigadoras efectuaron una inmersión profunda con la población, con el propósito de exponer los principales hallazgos y de que los participantes priorizaran las temáticas de su interés para cuyo abordaje debía ofrecerse propuestas metodológicas e indagar su percepción acerca del rol de enfermería dentro de un centro educativo.

Para el análisis de los resultados se aplicó la triangulación, una técnica que permite comparar las realidades de los adolescentes, el criterio profesional de las investigadoras y el referente teórico acerca de lo que es su adolescencia intermedia. El análisis de los datos fue guiado por una categorización de las unidades de análisis descritas anteriormente.

\section{Consideraciones éticas.}

A lo largo del proceso investigativo, se tomó en cuenta principios bioéticos como la beneficencia, no maleficencia, autonomía y justicia; por consiguiente, se diseñó un consentimiento informado con el que la persona encargada autoriza al adolescente a participar en el estudio. Asimismo, se utilizó un asentimiento informado dirigido a la persona adolescente para registrar su anuencia a formar parte de la investigación.

Previo a la identificación de necesidades, hubo una etapa de coordinación mediante la que se pretendió encontrar apoyo y autorización por parte de las entidades que estarían inmersas en el estudio. En primer lugar, se obtuvo el aval por parte de la directora del centro educativo, a lo que se aúna la colaboración de la Caja Costarricense del Seguro Social en diferentes niveles de atención, así como el respaldo del Programa de Posgrado de la Escuela de Enfermería.

\section{RESULTADOS}

Respecto de dónde viven, de los 32 adolescentes que participaron solo uno de ellos reside en la provincia de Alajuela y el resto habita en diferentes lugares de la provincia de Heredia.

En relación con el tipo de familia a la que pertenece cada adolescente, 20 conviven en familias nucleares convencionales, siete en monoparental, uno en familia monoparental extendida y cuatro en familias nucleares extendidas.

La población adolescente reconoció estar cursando la etapa de adolescencia, percibida como "una etapa difícil, llena de cambios, de muchas emociones encontradas" (grupo focal 8M), y "la edad que viene después de la niñez" (grupo Focal 8H) o "es una etapa como intermedia, de transición” (grupo focal 8M).

Con respecto a los gustos e intereses, la población recalcó que disfruta compartir con los amigos, lo cual se colige de comentarios como "salir con los amigos" (grupo focal 8M) y "conocer nuevas personas y tener nuevos amigos y amigas porque con ellos se tiene con quien compartir lo que piensan y sienten” (grupo focal 7H). 


\section{Revista Electrónica Enfermeria Actual en costa Rica}

Además, consideraron relevante el "cambiar un poco la actitud con los papás" (grupo focal 8M), incluso pretender "darle más importancia a las cosas...la familia" (grupo focal 7M), interesarse por estudiar y mantener o mejorar el rendimiento académico: "sacar mejores notas" (grupo focal 8M).

Por otra parte, la población se refirió a los cambios característicos de esta etapa, al expresar que "es una etapa de cambios y de aprendizaje" (grupo focal 7M); además de que reconocieron cambios físicos, emocionales y de madurez, lo cual se evidencia en frases alusivas a "la pubertad...la primera menstruación, el crecimiento y los cambios hormonales" (grupo focal $8 \mathrm{M}$ ); "cambios emocionales... a veces uno está bien y de pronto no, se pone mal sin saber por qué" (grupo focal $8 \mathrm{M}$ ); "cuando usted está niño, prácticamente no toma decisiones, sus papás toman las decisiones por usted y ya ahora más grande usted sabe si le gusta o no y qué es lo que quiere" (grupo focal 8M).

Por otro lado, los adolescentes describieron los rasgos que caracterizan las relaciones en cada una de sus familias, entre los cuales se discutieron los lazos de confianza que guardan con sus padres, madres, hermanos o hermanas, las reglas que usan los individuos para normar la convivencia, así como las diferencias esperables en el sistema familiar, acerca de lo que expresaron lo siguiente: "me tratan como una adulta y otras veces como una niña entonces para uno es medio confuso, o sea para algunas cosas sí es grande y para otras no" (grupo focal 8M) y "la confianza que los papás le dan para dejarlo salir a algún lado, bueno obvio que si usted se jala tortas no lo van a dejar ir ni a la esquina" (grupo focal 7M).

Con base en lo planteado por los jóvenes, se concluye que el apoyo que ellos esperan por parte de su familia incluye dos necesidades fundamentales, la primera de ellas corresponde a una ayuda en cuanto a recursos económicos y materiales, mientras que la segunda se refiere a un soporte emocional de parte de familiares que los aconsejan y orientan en diferentes situaciones.

En cuanto al ámbito académico, los adolescentes mostraron interés y agrado por asistir al colegio puesto que "es un lugar donde nos sentimos felices, porque se está con los amigos, se comparte, se vacila, se divierte...se pasa bien y se aprende" (grupo focal $8 \mathrm{M}$ ). Dicho interés se basa en las relaciones que mantienen con sus pares, mas no en el apoyo de sus docentes, argumento que se confirmó durante la observación, ya que las relaciones entre jóvenes y docente se limita a asuntos académicos. Acerca del tema, es importante señalar que la comunicación en dirección docente-estudiante busca resaltar la autoridad sobre los adolescentes, mientras que en sentido estudiante-docente se observa respeto y obediencia ante instrucciones; además, cabe mencionar que la institución educativa considera que responde a los gustos, intereses y necesidades de la población joven mediante las características físicas, al ser un lugar amplio, un espacio seguro, donde se observan diversas zonas de entretenimiento y alimentación, y es adecuado para propiciar el desarrollo de las actividades académicas.

Otra de las áreas discutidas en los grupos focales, fueron las características de las relaciones entre pares y su impacto a lo largo del desarrollo de la persona adolescente. Los pares en su mayoría corresponden a los compañeros del colegio o, bien, a personas que viven cerca de sus hogares, lo cual se ejemplifica con afirmaciones como "prefiero hablar con los amigos para hablar de ciertas cosas" (grupo focal 7H), "yo le cuento todo" (grupo focal 7M) y "jugar bola o ir a dar vueltas con los amigos" (grupo focal 7H). De igual manera, se encontró que la mayoría de la población adolescente ha establecido o mantiene alguna relación de pareja. 


\section{Revista Electrónica Enfermeria Actual en costa Rica}

Durante el espacio en el que se abordó las problemáticas comunes que experimentan, se identificó el fenómeno de las drogas, la violencia y situaciones relacionadas con la sexualidad, reflejado en afirmaciones como "muchas personas se adelantan a tener relaciones sexuales" (grupo focal $8 \mathrm{M}$ ); "nos inducen a consumir drogas: a mí me ofrecieron en un baile del cole" (grupo focal $8 \mathrm{H}$ ), y "la violencia o cuando alguien se aprovecha de otra persona" (grupo focal 7H).

Posterior a la discusión analizada, las investigadoras procedieron a preguntarles qué esperarían que haga una enfermera o enfermero dentro de un colegio, con el objetivo de identificar sus necesidades y deseos, así como el conocimiento que tengan de la labor que podría desempeñar un profesional de salud. Las expresiones más comunes giraron alrededor de dos funciones: brindar cuidado directo: "si alguien se pone mal que ustedes lo atiendan, que tengan pastillas, ante alguna emergencia" (grupo focal $8 \mathrm{H}$ ), y acompañamiento: "yo creo que debería ser una persona con la que uno pueda hablar de diferentes cosas, o si uno tiene un problema y ella la puede escuchar y ser como una amiga" (grupo focal 8M).

En algunos casos, los participantes señalaron la importancia de contar con un profesional a cargo del curso de afectividad y sexualidad, el cual actualmente es impartido por los docentes, al respecto mencionaron: "darnos información como de sexualidad, porque el curso que hay es muy aburrido" (grupo focal 7H), "porque el curso de sexualidad nadie lo toma en serio" (grupo focal 8M) y "es que se han centrado en hablar de impulsos y no explican nada" (grupo focal $8 H$ ).

Una vez obtenidos los datos de los adolescentes que participaron a lo largo de este primer momento de identificación de necesidades (sexualidad, drogas, violencia), se realizó un segundo acercamiento con el fin de confirmar los hallazgos a través de los mismos grupos de discusión.

Uno de los puntos desarrollados en la discusión fue el de priorizar las temáticas según el orden de importancia mencionado por los estudiantes, acerca de lo que se encontró que el fenómeno de las drogas y situaciones relacionadas con la sexualidad ocupan los dos primeros lugares, seguidos por violencia. Este último tema no se abordó, ya que desde la perspectiva de la población, no representaba una problemática frecuente en su cotidianeidad.

Una vez ordenados según grado de relevancia, se les solicitó detallar cuáles serían los tópicos por desarrollar dentro de cada una de las problemáticas. Con respecto a la temática de drogas, manifestaron relevante incluir las razones de consumo, las consecuencias, los efectos de las drogas en el cuerpo y cómo prevenir el consumo. En cuanto al tema de la sexualidad en la persona adolescente, destacan las razones por las que una persona decide tener relaciones sexuales, conocimientos sobre métodos anticonceptivos, prevención del embarazo y enfermedades de transmisión sexual, entre otros.

En relación con las metodologías sugeridas por los participantes para fortalecer su conocimiento según las temáticas identificadas, se detectó que consideran importante hacerlo en un ambiente de confianza desde estrategias participativas, en las que desarrollen actividades de forma grupal, e individuales, si requirieran mayor privacidad, profundidad e intimidad.

\section{DISCUSIÓN}




\section{Revista Electrónica Enfermeria Actual en costa Rica}

La razón por la que se realizaron grupos focales del mismo sexo correspondió a que durante la etapa intermedia de la adolescencia se conoce que ese tipo de grupos generan un espacio de mayor confianza en comparación con los de sexo opuesto, en los que los adolescentes muestran su interés por comprobar su capacidad de atraer al otro (Casas y Ceñal, 2005). De forma que al discutirse temáticas que abarcan sus percepciones, experiencias y emociones, fue indispensable que se sintieran con mayor libertad y seguridad.

El concepto de esta etapa de la vida coincide con lo planteado por Blanco et al (2011), para quienes la adolescencia es un periodo de transición entre la niñez y la adultez o una etapa particular de la vida humana. En cuanto a los cambios propios de esta etapa, los y las participantes coinciden con lo establecido por Retana (2007), al indicar que la adolescencia es el comienzo de cambios biológicos, psicológicos y sociales.

En el ámbito de las relaciones interpersonales, Retana (2007) y González (2010) exponen que a esta edad hay una preocupación más evidente por la afirmación y aceptación personal y social, así como la necesidad de integrar en la vida cotidiana nuevas actividades para interaccionar con el grupo de pares, lo cual concuerda con lo mencionado por los adolescentes como algo primordial en sus vidas. Respecto de lo anterior, que la población identifique a los pares como personas importantes en esta fase de la vida, permite reconocerlos como un factor de protección, debido a que se percibe un carácter positivo y amistoso por los pares, además de una buena relación, habilidades sociales y el respeto a los derechos y necesidades del otro (Zubarew, 2013).

Una de las vivencias necesarias en la adolescencia es la formación de grupos heterosexuales. De manera que la separación según sexo, propia de la infancia, ha sido superada para convivir con personas del sexo opuesto mediante el disfrute de actividades similares (Retana 2007, y González, 2010), como ir a fiestas, al cine, a pasear por el parque, entre otros. Desde esa misma línea de pensamiento de los autores citados, cabe mencionar que durante la adolescencia los jóvenes buscan diferenciarse del grupo familiar. En los grupos focales se planteó el deseo tanto de experimentar vivencias en un ámbito extrafamiliar, como en lo referente a mantener relaciones sin grandes conflictos con sus familias; por tanto, la familia funciona como otro factor protector, idea reforzada por Zubarew (2013), quien afirma que la constitución de un sistema de valores proporcionado por la familia permite a la persona poseer sentimientos como la esperanza, alegría, éxito y amor, mientras que el distanciamiento o la ausencia de una relación con los padres o madres condiciona a la persona adolescente ante un riesgo a lo largo de su adolescencia.

En relación con lo anterior, uno de los requisitos en la familia con adolescentes se relaciona con modificar la relación padres/madres-hijos/hijas de manera que se les permita entrar y salir del sistema (․ㅡodríguez, 2013); es decir, se trata de una mayor flexibilidad en los límites establecidos, con el fin de permitir un enlace con la sociedad (Satir, 2005). Al respecto, también se le da importancia al despliegue de confianza como posibilidad o libertad para compartir sus asuntos, necesidades y el pedir o dar consejos.

Otra de las situaciones que exponen los participantes corresponde a lo denominado por Rodríguez (2013), "la existencia de un intervalo de cuatro años en la adolescencia", en el que los padres y las madres perciben a sus hijos o hijas dos años más jóvenes de lo que realmente son, mientras que la persona adolescente, por el contrario, se considera dos años mayores de su edad cronológica.

Respecto de los quehaceres que realizan, llama la atención la diferencia descrita entre los quehaceres 


\section{Revista Electrónica Enfermeria Actual en costa Rica}

manifestados por los hombres en comparación con las mujeres, ya que hacen alusión a una construcción social de masculinidad y feminidad dentro de un sistema patriarcal, donde los hombres principalmente tienen aspiraciones económicas y académicas, mientras que las mujeres se enfocan en situaciones relacionadas con el hogar y su afectividad (Rodríguez-Araya, 2013).

El tercer factor de protección identificado fue el establecimiento de las metas educacionales y vocacionales, ya que la población participante considera relevante mantener o mejorar su rendimiento académico, comportamiento que según Retana (2007) y González (2010), es común en la adolescencia, etapa en la que se explora las capacidades personales, entre las que se incluye las habilidades de formación académica. En torno al tema, se añade que el entorno académico cobra un gran significado durante la etapa de la adolescencia debido a que, según exponen Jiménez y Gaete (2013), es un espacio social que satisface parte de las necesidades afectivas, de pertenencia e identidad, además de que, como agrega Krauskopf (2000), la educación se constituye en un ambiente esencial para la formación social y ciudadana de los individuos y grupos.

El proceso de crecimiento y desarrollo de la persona adolescente en etapa intermedia implica multiplicidad de condiciones y situaciones, las cuales interaccionan entre sí para generar cambios significativos en los adolescentes (Villegas, 2009). Fue posible apreciar que la población poseía conocimientos acerca de los tipos de cambios esperados durante la adolescencia, entre los que se hallan, según Cattani (s.f.), los físicos puesto que son los estrógenos y la testosterona los responsables del incremento longitudinal, el cambio de las proporciones corporales, la distribución de la grasa, el cambio de la voz, la aparición de caracteres sexuales secundarios y el desarrollo muscular característico de esta etapa de la vida. Sobre el tema, después de triangular los hallazgos con la teoría sobre los cambios emocionales experimentados por los jóvenes, es posible afirmar la existencia de una crisis de identidad para esta etapa, la cual consiste en la necesidad que posee la persona adolescente de ser ella misma, de definir su yo, sus objetos y de adquirir algo que la diferencie de la niñez y la adultez a lo que se debe sumar una marcada labilidad e hiperreactividad emocional expresada en comportamientos incoherentes e impulsivos, explosiones afectivas intensas, pero a la vez superficiales. (Zubarew y Gumucio, 2013).

Entre otros cambios relevantes para la población se destaca los relacionados con la afirmación del atractivo sexual y social, que evidencian los emergentes impulsos sexuales, así como el deseo de experimentar vivencias relacionadas con el amor (Retana, 2007; González, 2010).

En cuanto al último bloque de cambios, correspondientes a aumentar cierto grado de madurez, Vygotsky plantea que responden al desarrollo cognoscitivo de la persona y se vinculan con la toma de decisiones, el pensamiento crítico y creativo, estadio en el que también los jóvenes alcanzan una mayor comprensión de las situaciones y de la idea de causa-efecto (Villegas 2009).

Una vez analizados los cambios e intereses propios de la adolescencia, se inició la discusión en torno a las problemáticas que los adolescentes enfrentan a lo largo de esta etapa. La primera de ellas fue la problemática del fenómeno de las drogas, sobre la que Vargas (2009) afirma que toma importancia y genera mayor riesgo, debido a las características propias de este periodo de la vida en el que suele empezar el consumo de bebidas alcohólicas y otras drogas (a los 13 años aproximadamente).

Tal como se mencionó, el característico aumento de la secreción hormonal en esta edad aumenta la 


\section{Revista Electrónica Enfermeria Actual en costa Rica}

atracción por el sexo opuesto o el mismo sexo y se manifiesta precocidad de las relaciones sexuales, lo cual provoca un incremento en los embarazos adolescentes, contagio de infecciones de transmisión sexual, entre otros (Shutt-Aine y Maddaleno, 2003), razón por la que es imprescindible que el abordaje de tales temas sea de una manera abierta, entretenida y acorde con sus necesidades de conocimiento.

Es posible vincular los diferentes elementos discutidos con los sistemas personal, interpersonal y social planteados por Imogene King: el primero corresponde a las apreciaciones expuestas por parte de los adolescentes, las cuales facilitan al profesional de enfermería comprenderlos y construir con ellos metas encaminadas al mejoramiento de su salud. El segundo se evidencia en las diferentes relaciones y los roles que mantiene el adolescente, ya sea con su familia, pares, autoridad, mientras que en el tercero, se observa la influencia del sistema social, puesto que además de que la persona interacciona con su medio y con otros individuos para el logro de objetivos específicos, existe un sistema organizado de límites, de roles sociales, prácticas y normas para mantener valores y mecanismos de regulación (King, 1984).

Dado que durante los grupos focales se solicitó recomendaciones metodológicas para el abordaje de las necesidades identificadas en la población, fue pertinente realizar la triangulación teórica con lo descrito, con el fin de brindar una intervención idónea de la persona adolescente. En relación con lo mencionado, Breinbauer y Maddaleno (2008) recalcan que el desafío de las personas que trabajan y conviven con jóvenes es demostrar un interés activo para mejorar su salud, y apoyarlos de la mejor manera posible para propiciar en ellos la capacidad de ser autónomos y conscientes en la toma de decisiones.

En síntesis, los profesionales de enfermería pediátrica poseen el conocimiento requerido para desarrollar un programa de atención de la persona adolescente en sus diferentes ámbitos y frente a cada una de sus necesidades. Dicho programa debe abarcar una entrevista individual para la persona adolescente, en la que se considere sus preocupaciones, se evalúe e indague en función de la etapa de la adolescencia e implemente actividades grupales para el abordaje de las necesidades primordiales para los adolescentes, a saber prevención del consumo de drogas y sexualidad segura, de modo que los especialistas articulen las distintas esferas en las que está inmersa dicha población.

\section{Conclusiones}

Se demuestra la utilidad de establecer un primer contacto con los adolescentes en función de identificar de forma conjunta sus necesidades existentes y brindarles la oportunidad de proponer posibles respuestas. El acercamiento es una herramienta que favorece el establecimiento de la empatía y una mayor apertura del individuo frente a los profesionales de enfermería.

El uso de la teoría de Imogene King funge como una guía para identificar e interpretar las necesidades existentes en la población adolescente, incluyendo diversos componentes de los sistemas para valorar a la persona adolescente desde sus diferentes áreas del desarrollo y esclarecer el rol del profesional en función del alcance de los objetivos.

El formular un diagnóstico de necesidades dentro del escenario educativo propicia una mayor apertura por parte de los adolescentes, puesto que contribuye a fortalecer la confianza, además de que permite observar su 


\section{Revista Electrónica Enfermeria Actual en costa Rica}

desenvolvimiento en un entorno cotidiano que promueve el empoderamiento de su posición, opiniones, pensamientos y decisiones.

Las principales necesidades de la población adolescente del Colegio Ing. Samuel Sáenz Flores aluden a su experiencia durante esta etapa de la vida, la importancia de las relaciones interpersonales, y las principales problemáticas a las que se exponen: el consumo de drogas, la sexualidad y, en menor grado, la situación de violencia entre pares.

Es posible afirmar que las personas adolescentes que asisten a sétimo y octavo grado del Colegio Ing. Samuel Sáenz Flores manifiestan la necesidad de desarrollar un programa de enfermería pediátrica para la atención de temáticas relacionadas con el consumo de drogas y sexualidad.

Los profesionales de enfermería pediátrica que pretendan acompañar a la persona adolescente durante esta etapa de la vida, deben escuchar atentamente su voz y articular el conocimiento adquirido a partir de las diferencias individuales, a saber cultura, género, sistema familiar, entorno en el que se desenvuelve, experiencias previas, condición socioeconómica, relaciones personales, percepción y necesidades.

\section{REFERENCIAS BIBLIOGRÁFICAS}

Asamblea Legislativa de la República de Costa Rica. (2001). Código de la Niñez y la Adolescencia. Costa Rica.

Blanco, M., Jordán, M., Pachón, L., Sánchez, T., y Medina, R. (2011). Educación para la salud integral del adolescente a través de promotores pares. Rev Méd Electrón , 33 (3), 349-359.

Breinbauer, C., y Maddaleno, M. (2008). Jóvenes: opciones y cambios. Promoción de conductas saludables en los adolescentes. Whashington D.C.: OPS-OMS.

Caja Costarricense del Seguro Social. (2012). Evaluación del desempeño de la prestación de servicios de salud. Informe de resultados 2011. San José: CCSS.

Campbell, N., Tuttle, J., Knapp, T. (2009). The Effect of Positive Adolescent Life Skills Training on Long Term Outcomes for High-Risk Teens. Journal of Addictions Nursing , 20, 6 - 15.

Casas, J., Ceñal, J. (2005). Desarrollo del adolescente. Aspectos físicos, psicológicos y sociales. Pediatría Integral , $9(1), 20-24$.

Cattani, A. (s.f.). Crecimiento y Desarrollo del Adolescente. Lección 1: Crecimiento y Desarrollo Puberal durante la adolescencia. Chile: Pontificia Universidad Católica de Chile.

Fondo de las Naciones Unidas para la Infancia. (2011). Estado Mundial de la Infancia. La adolescencia una época de oportunidades. Nueva York: UNICEF.

González, M. (2010). Análisis del aporte de la creación de la consulta de Enfermería Pediátrica para los niños, 
niñas y preadolescentes de los Centros Infantiles de la Asociación Roblealto en el año 2009. San José, Costa Rica: Universidad de Costa Rica.

Hernández, R., Fernández, C., y Baptista, P. (2010). Metodología de investigación (5ta ed.). México: McGraw-Hill.

Instituto Nacional de Estadística y Censos. (2011). Costa Rica: Población total por grupos de edad, según provincia, cantón y sexo. Recuperado el 15 de mayo de 2013, de http://www.inec.go.cr/Web/Home/GeneradorPagina.aspx

Jiménez, W., y Gaete, M. (2013). Estudio de la exclusión educativa y abandono en la enseñanza secundaria en algunas instituciones públicas de Costa Rica. Revista Electrónica EDUCARE , 17 (1), 105-128.

Jolly, K., Weiss, J., Liehr, P. (2007). Understanding adolescent voice as a guide for nursing practice and research. Issues in Comprehensive Pediatric Nursing , 3-13.

King, I. (1984). Enfermería como profesión. Filosofia, principios y objetivos. México, D.F.: LIMUSA.

Krause, M. (1995). La investigación cualitativa. Un campo de posibilidades y desafíos. Temas de Educación (7), 118.

Krauskopf, D. (2000). Participación social y desarrollo en la adolescencia. San José, Costa Rica: UNFPA.

Martínez, D. et al. (2013). Intervención de Enfermería en la adolescencia; experiencia en una institución de estudios secundarios pública. Enfermería Actual en Costa Rica (24).

Muñoz, D., Argüello, R., Arias, M., Gómez, G., y SilesM. (2009). Programa de Enfermería sobre educación en salud dirigido a los y las adolescentes de educación diversificada especial del Colegio Técnico Industrial de Calle Blancos. Enfermería Actual en Costa Rica (17).

Organización de Naciones Unidas. (1989). Convención sobre los derechos del niño.

Organización Panamericana de la Salud. (2007). Renovación de la atención primaria de salud en las Américas. Washington DC.: OPS/OMS.

PANI- UNICEF. (2009). Política Nacional para la Niñez y la Adolescencia Costa Rica 2009-2021. Costa Rica.

Parvizy, S., y Ahmadi, F. (2009). A qualitative study on adolescence, health and family. Mental Health in Family Medicine, 163-172.

Programa de Posgrado en Ciencias de la Enfermería. (s.f.). Perfil del graduado de la Maestría en Enfermería Pediátrica con énfasis en Niñez y Adolescencia. San José: Universidad de Costa Rica.

Retana, M. (2007). Formas en que se puede manifestar y reproducir conductas violentas hacia la pareja en las 
relaciones de noviazgo. San José, Costa Rica: Universidad de Costa Rica.

Rew, N., Horner, S., y Brown, A. (2011). Health-risk behaviors in early adolescence. Issues in Comprehensive Pediatric Nursing , 34, 79-96.

Rodríguez, A. (2013). Unidad II: La familia como unidad vital de apoyo y contención en el contexto social. En G. Casas, Qué se diagnostica en un sistema familiar. San José: Universidad de Costa Rica.

Satir, V. (2005). Nuevas Relaciones Humanas En El Núcleo Familiar . México D.F.: Editorial Pax.

Shutt-Aine, J., y Maddaleno, M. (2003). Salud sexual y desarrollo de adolescentes y jóvenes en las Américas: Implicaciones en programas y políticas. Washington, DC: Organización Panamericana de la Salud.

Vargas, Z. (2009). La Investigación aplicada: una forma de conocer las realidades con evidencia científica. Revista Educación, 33 (1), 155-165.

Villegas, I. (2009). Programa para el desarrollo de habilidades cognitivas, conductuales y emocionales para las relaciones inter e intrapersonales en niños, niñas y adolescentes albergados en la Aldea Arthur Gough. San José: Universidad de Costa Rica.

Zubarew, T. (2013). Evaluación Clínica del Adolescente. Supervisión de Salud del Adolescente. Lección 2. parte 1. Chile: Pontificia Universidad Católica de Chile.

Zubarew, T., Gumucio, M. (2013). Crecimiento y Desarrollo del Adolescente. Lección 2: Desarrollo psicosocial y psicosexual en la adolescencia. Chile: Pontificia Universidad Católica de Chile. 